\title{
A Million Crystal Structures: The Whole Is Greater than the Sum of Its Parts
}

Robin Taylor and Peter A. Wood*

Cambridge Crystallographic Data Centre, 12 Union Road, Cambridge CB2 1EZ, United Kingdom wood@ccdc.cam.ac.uk 


\section{Search: search3 (Thu Mar 07 15:45:51 2019): Hits 1-4}

DADNUR

Reference:

R.Fernandez de la Pradilla, C.Montero, J.Priego,
L.A.Martinez-Cruz (1998) J.Org.Chem. ,63,9612

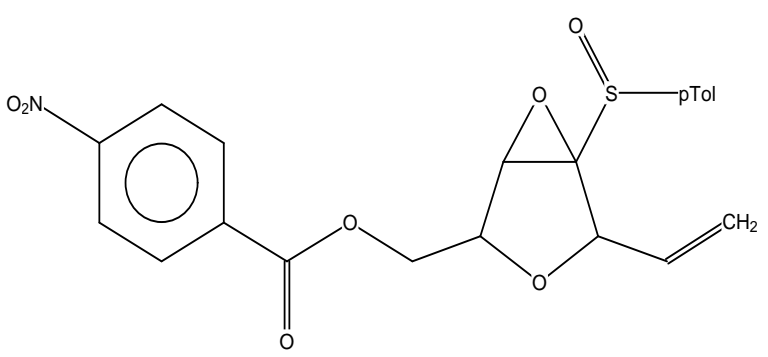

DCTXAN

Reference:

H.Shimanouchi, Y.Sasada (1979)

Acta Crystallogr.,Sect.B:Struct.Crystallogr.Cryst.Chem. ,35,1928

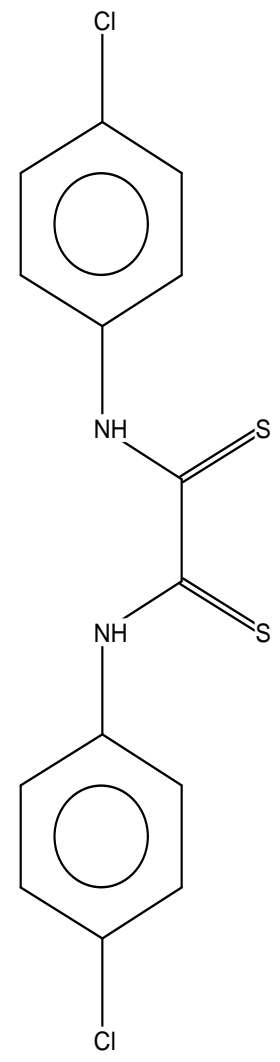

DAKVES

Reference: J.Lieffrig, H.M.Yamamoto, T.Kusamoto, Henbo Cui,

O.Jeannin, M.Fourmigue, R.Kato (2011) Cryst.Growth Des. ,11,4267

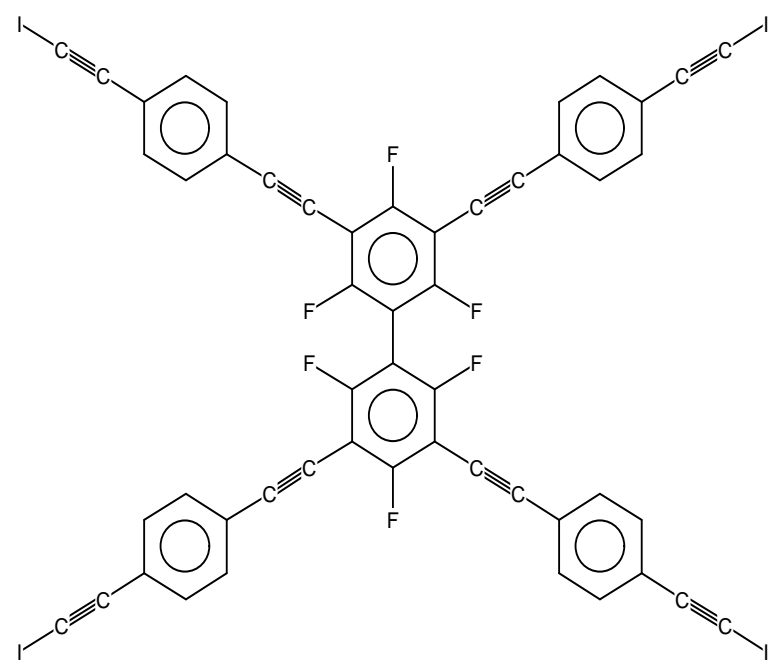<smiles>C1CSC2=C(SC1)SC(=C1SC3=C(SCCCS3)[SH+]1)S2</smiles>

$\mathrm{Cl}$

\section{FUQGAZ}

Reference:

T.D.Avery, G.D.Fallon, D.K.Taylor, E.R.T.Tiekink (2000) Z.Kristallogr.-New Cryst.Struct. 215,257

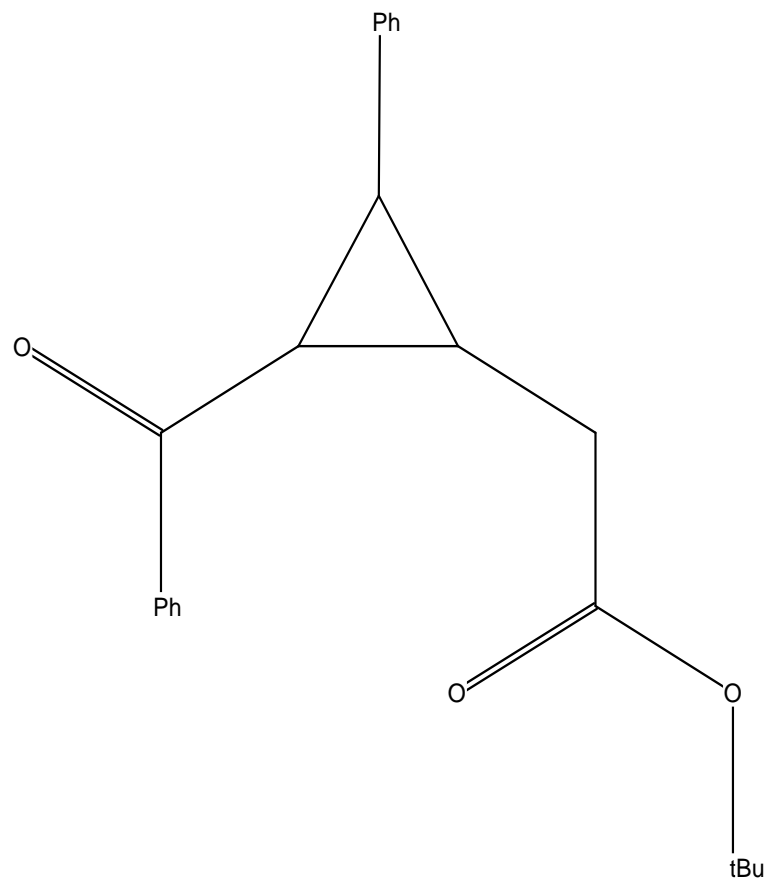




\section{Search: search3 (Thu Mar 07 15:45:51 2019): Hits 5-8}

FUYBUX

Reference:

Yong-Biao Men, Junliang Sun, Zhi-Tang Huang, Qi-Yu Zheng (2010) Chem.Commun. ,46,6299
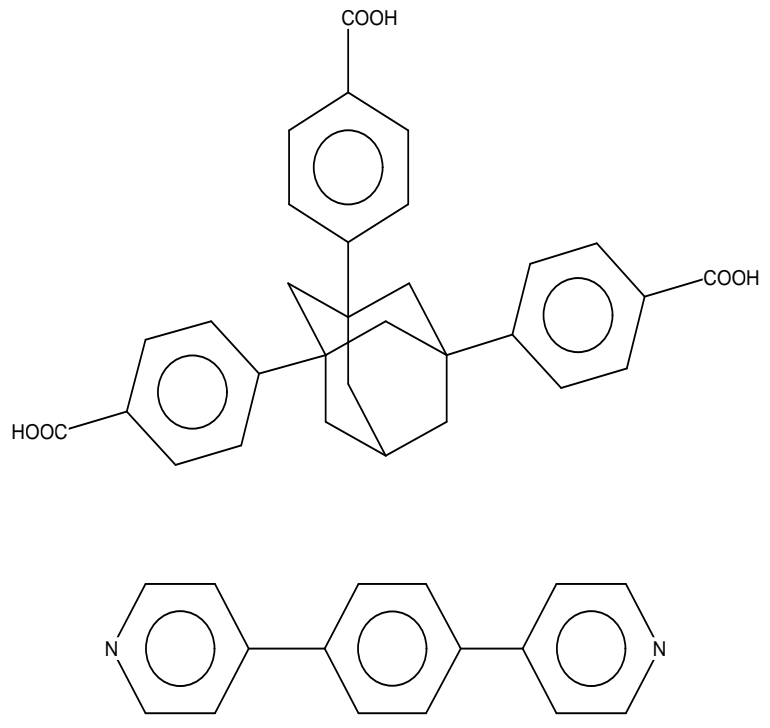

$\mathrm{HO}-\mathrm{CH}_{3}$

NAHCOQ

Reference:

C.Mukhopadhyay, S.Rana, R.J.Butcher (2010) ARKIVOC,

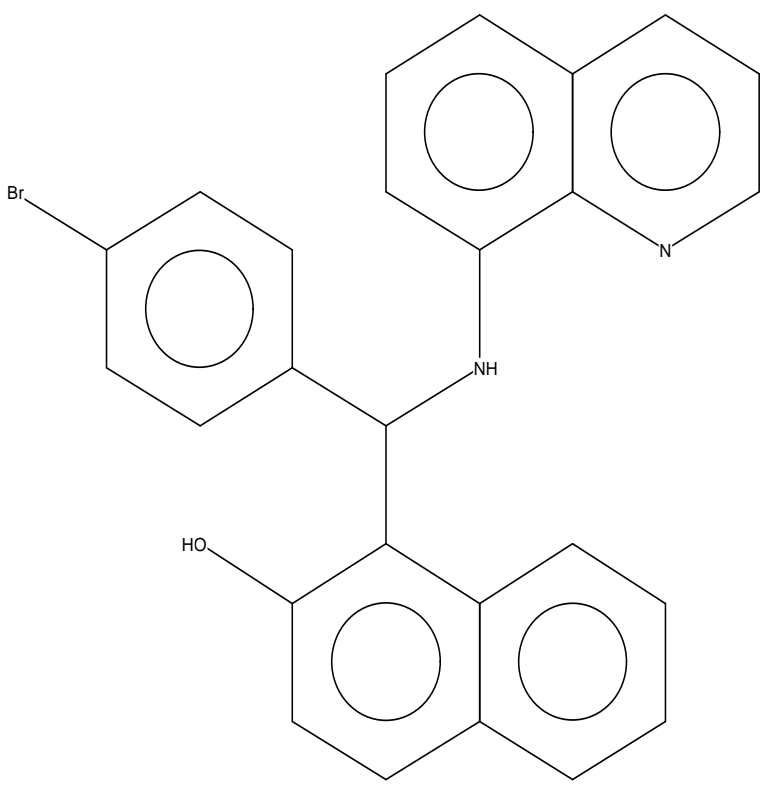

GEDLEH

Reference:

A.R.Katritzky, Hongfang Yang, Dazhi Zhang, K.Kirichenko, M.Smiglak, J.D.Holbrey, W.M.Reichert, R.D.Rogers (2006) Now J 30,349

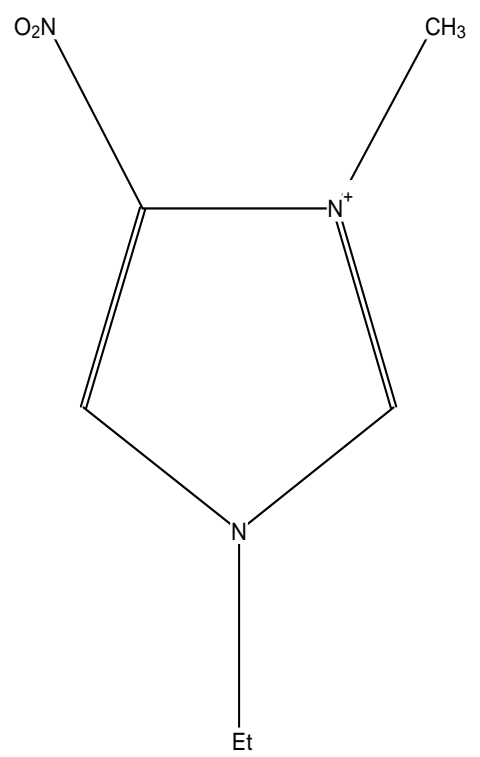

$\mathrm{F}_{3} \mathrm{C} \longrightarrow \mathrm{SO}_{3}^{-}$

OFATAT

Reference: Liang Zhang, A.J.Stephens, A.L.Nussbaumer,

J.-F.Lemonnier, Pia Jurcek, I.J.Vitorica-Yrezabal, D.A.Leigh (2018) Nature Chemistry, 


\section{Search: search3 (Thu Mar 07 15:45:51 2019): Hits 9-12}

PORPIN02

Reference:

I.Saltsman, I.Goldberg, Y. Balazs, Z.Gross (2007)
Tetrahedron Lett. , 48,239

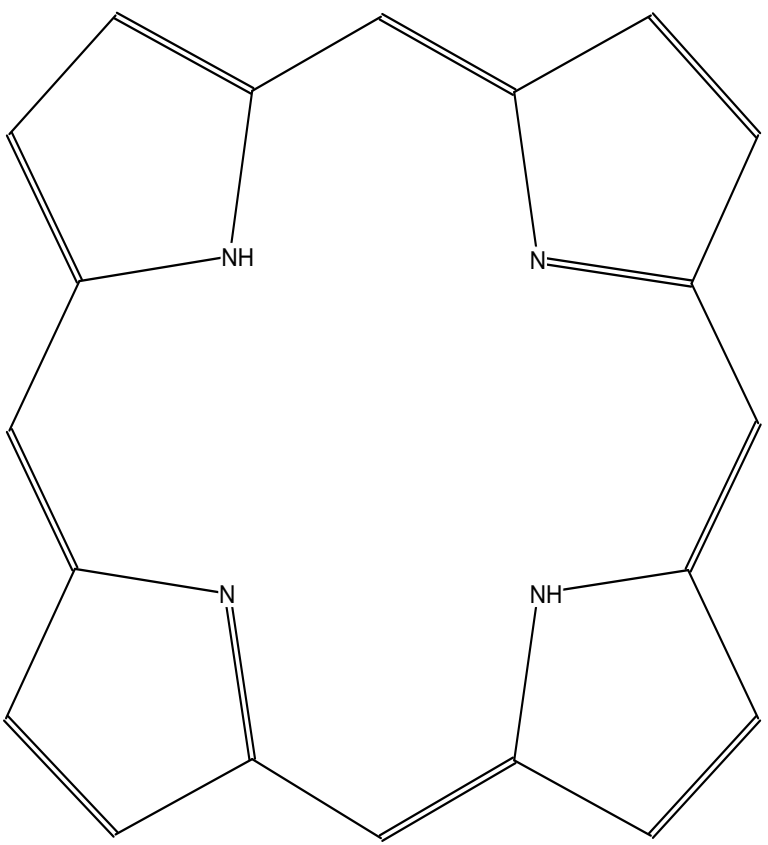

TETXUL01

Reference:

S.Sarkar, M.S.Pavan, T.N.Guru Row (2015) Phys. Chem.Chem.Phys.(PCCP) ,17,2330

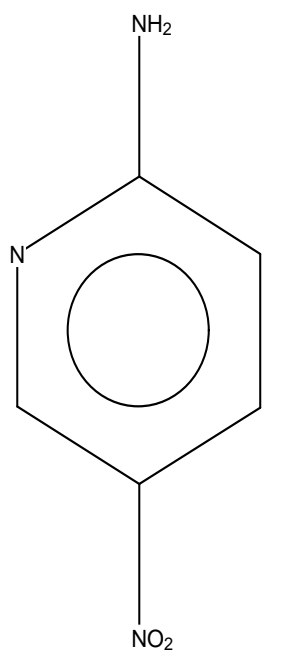

SIZPIB

Reference:

A.Takahashi, H.Yanai, T.Taguchi (2008) Chem.Commun.

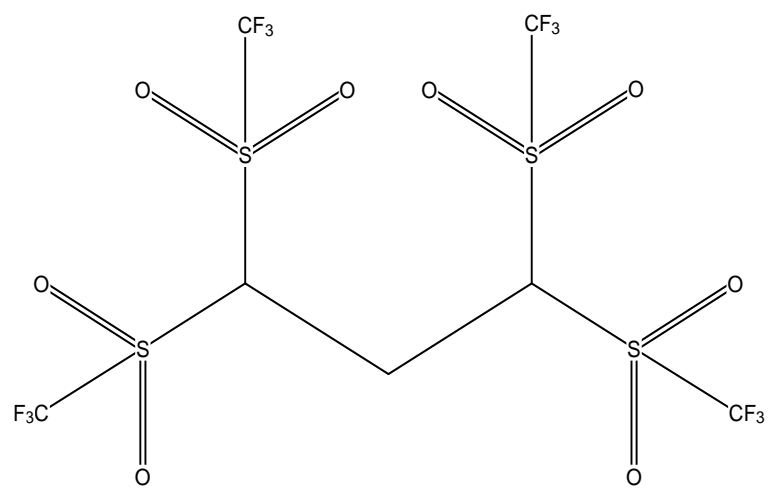

UHECAL

Reference:

Deyuan Kong, Xiaoying Huang, Yuyuan Xie (2002) Inorg.Chim.Acta ,340,133

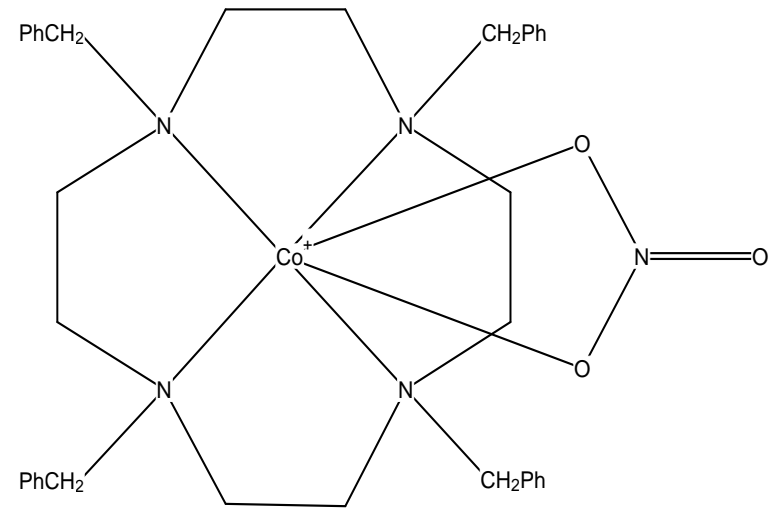


Search: search3 (Thu Mar 07 15:45:51 2019): Hits 13-16

VIHNEF

Reference:

J.Bittner, K.Seppelt (1991) Chem.Ber. ,124,87

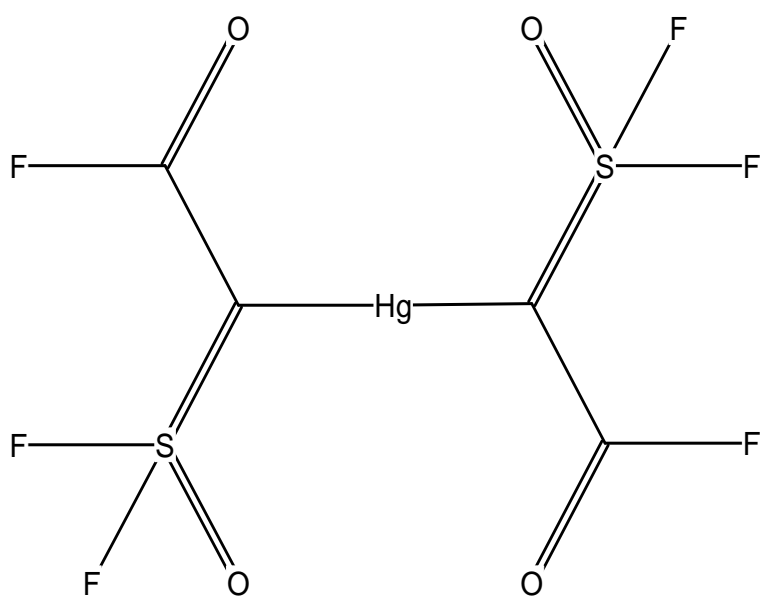

ZAQCOL01

Reference:

S.P.Thomas, M.S.Pavan, T.N.Guru Row (2014) Chem.Commun. ,50,49

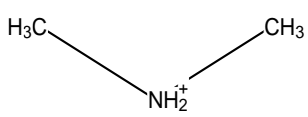

WILWUJ

Reference: $\quad$ C.Rudinger, H.Beruda, H.Schmidbaur (1994)

Z.Naturforsch., B:Chem.Sci. 49,1348

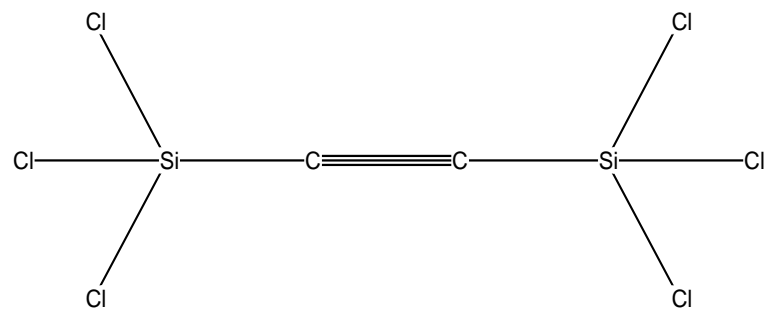

ZEKPOW02

Reference:

S.P.Thomas, M.S.Pavan, T.N.Guru Row (2014) Chem.Commun. 50,49

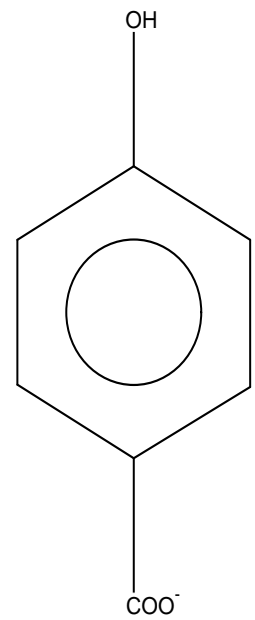

